\title{
Plant and Its Control Mechanisms
}

\author{
Martin Vlček \\ eFunctionality, non-profit research group, Prague 18200, Czech Republic
}

Received: April 5, 2015 / Accepted: April 29, 2015 / Published: April 30, 2015.

\begin{abstract}
The regeneration of its cells is one of the two goals of the plant. The control systems try to allow to cells to optimize their state depending on the circumstances, sometimes in advance. The first of these systems is located in the root. It controls the materialistic input for the regeneration and at the same time is the source of primary information about the state of this input and its development at time. Another element of the control is the system controlling the formation and the distribution of the energy. This control is made without making provision for the state of individual cells. The successfulness of individual cells is taking into account by the system connected with companion cells in the vascular bundle. The similar holds for the second system. It, on the basis of the tension on the boundary of the plant, controls the dynamics of the boundary and removes the tension. The second goal of the control, besides the regeneration, is the optimization of the existence of the plant as whole. For this sake new global criteria arise and are used throughout the control.
\end{abstract}

Key words: Plant, tension, boundary, control, successfulness.

\section{Introduction}

The basic properties of every object are derived from the functioning of its control systems. These systems function on different levels with the same logic, even if they are working with different elements. However, goals and means are the same [1-4].

The present work also arises from this logic. It uses findings from analogical systems proved by extended calculations [5-8] and finds and applies them in the plant.

On the other hand, the author realizes that, similarly as with the mentioned objects, it is necessary to prove them experimentally in the plant as well.

\section{The Control of the Plant}

\subsection{The Central Axe - the Regeneration}

The basic function of the plant, its central axe, is the regeneration of cells, besides the control of the existence of the plant as whole. It consists not only in the solution of sequels of activities of cells or changes

Corresponding author: Martin Vlček, Dr., research field: cybernetics. E-mail: martin.vlcek@efunctionality.eu. in the environment, but also in a preparation to them, occasionally in an avoidance of undesirable states.

The regeneration proceeds on two levels - on the level of the plant as whole and on the lower level-insides cells and consists in the delivery of consumed materials or in the redistribution of them. On the other side, it is necessary to remove some products of the metabolism, what for new proceeds on different levels.

\subsection{The Control of the Regeneration}

At the beginning of the system of the regeneration stays the system of the root as a main supplier of materialistic inputs. It is the source of basic stimuli for the following control. These stimuli input into the phloem of the root by the way of companion cells.

It seems that the companion cells are the global place of control systems. Firstly, because of their position between the phloem and the xylem in the vascular bundle, having the occasion to monitor the regeneration from two points of view-from the beginning and from the end. Secondly, because of the critical position at the end of the distribution of 
assimilation to final consumers, with the possibility to influence it. From now on individual types of companion cells will not be distinguished, because of the absence of relevant information. But their roles, their specializations are of course quite different.

The second, all embracing system is the control of the energy. This system controls the consumption and the deposit of the energy depending on the overall state of the regeneration. It stems from global indicators, evidently in phloem, and intervenes on some levels.

Around the central axe and its energetic control there are two other higher systems of control. They exist in all stabile objects in the nature. Both work with the successfulness of cells. The successfulness of individual cells in their work evaluates the system named EM0. Satisfaction of the plant as whole, stemming from the tension on the boundary and thus from the successfulness of cells in the boundary solves the system named as EM1.

\section{The Input System}

The input system VS represents a relatively independent and highly important element in the regulative structure of the plant. It consists anatomically of the rhizodermis, of a part of xylem and of accompanying cells between the phloem and the xylem in the root.

Besides the function connected with the absorption of the water and minerals this is the place of origin of initial information concerning the state of the regeneration, resp. its prepositions, it means about inputs being to the disposal in the soil in the environment. Impulses input from it through the pathway of accompanying cells into the part of phloem in the root and form a gradient in what work the control system EM1-the system for "the control of boundaries".

Thus there is a bifurcation of pathways in the input system. The materialistic way continues by the part of xylem into the above-ground part and after on to the

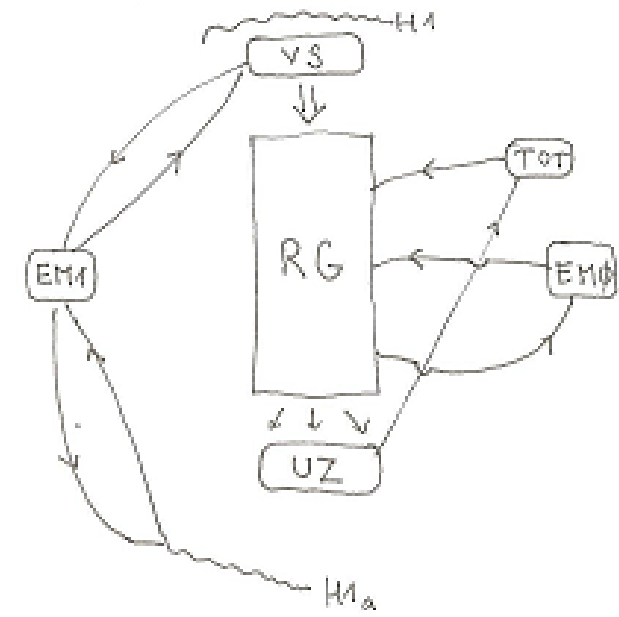

Fig. 1 Structure of the control of the plant.

VS-input system over the materialistic input into the regeneration RG. It is the main source of impulses for EM1, the system of control of the tension on the boundary of the plant. H1-1a-boundaries of the plant. UZ-consumers of the regeneration, the source of impulses for TOT, the system of the control of the regeneration through the flow of the energy. EM0 - the system of the control of the regeneration on the basis of the information about the successfulness from inside the process of the regeneration.

places of photosynthesis. The signal way inputs into accompanying cells and into the part of phloem and thus brings the information concerning the state of individual parts of the boundary.

\section{TOT}

The lowest level of the control is connected with the production and distribution of the energy. Its singularity is among others in the fact that it does not go out from the feedback from individual cells making regeneration, but from the overall level of the regeneration. This control system is named later on TOT.

Depending on circumstances its controls do not need to be realized by only one phytohormone but by a cooperation of some of them, with a certain role of e.g. cytokinin. Primary place of the origin of the signals is perhaps the parenchyma in the root, having access to the information from the phloem, from its terminal part, and at the same time having possibility to insert a carrier of information at the origin of the 
wooden part.

This system controls two levels connected with the energy - the level of the plant and the level inside the cells. It goes on the first level about the stimulation of parts of plant connected with the deposit of reserves, depending on the level of the global criterion in the phloem. The second level is the projection of this control inside cells and the influencing of organelles connected with the energy. It means mitochondria and plastids various types. This control inside cells controls the equilibrium between the deposition and the consumption of the energy.

Besides chemical agents, it is possible that TOT reacts also on a dynamics of some components in the phloem, when the volatility of their concentration is just the demonstration of a certain state of regeneration.

\section{EMO}

The task of EM0 is to make the plant a home of successfulness and thus of satisfied cells. It reaches it through the monitoring of the success of individual cells and on the basis of it controls the flow of the assimilation.

First of all, it is necessary to determine how the successfulness of a cell can be followed up. It reflects the level of participation into the system. Consumption or the production of the energy is one of possibilities and thus the concentration of the oxygen or of $\mathrm{CO}_{2}$ can be used as appropriate information. The process is as follows. The successfulness of a cell is evaluated by accompanying cells of the vascular bundle and to some extent is aggregated. The flow of the assimilation is disseminated from the phloem to its consumers on the basis of this evaluation, been appropriate to the successfulness.

The second effect of the successfulness is the fact, that the vascular bundle itself is more oriented into areas, where the successfulness is higher and inversely, the supply is suppressed in the area unsuccessfully. Thus it is a permanent reconstruction.
It seems in plant, that there is a control of flow already on the level of a cell, it means in the cytoplasm of accompanying cells. Not only the choice of plazmodezmat, but also the choice of other inner structures is controlled on the basis of the information about the successfulness.

\section{EM1}

\subsection{Principles}

The task of EM1 is to control the tension in the boundary. The boundary mainly concerns the rhizoidal part of the plant; it means the border between the inner environment and the environment of the water and of minerals. This boundary will be later on named as H1. After that, there is the boundary H1a, what is the border between the inner and outer environment in the sprout.

The tension on $\mathrm{H} 1$ rises in the case, when different parts of the root supply different necessary inputs, in spite of being relatively close to each other. In the terminology of the success, it goes around the situation, when a very successful part of rhizodermis is near to an unsuccessful one. And as near it is, as high is the tension. And the control and the reconstruction of the root stems on the basis of this tension.

Similarly is used the tension on H1a, where the difference mainly concerns the illumination of near parts.

Auxin is perhaps used in both cases as a working agent, in cooperation with other materials, e.g. cytokinin. Principle is as follows. Accompanying cells in the vascular bundle spread information concerning the tension toward the phloem, probably on filamentary structures, perhaps in the form of dynamical events on them. These events are after on used to the backward spreading of auxin to the place of the origin of the tension. Auxin continuously outputs from phloem and is controlled by the importance of the information, it means by the intensity of the remote tension. 


\subsection{The Place of Incidence}

The activity of the working agent is dual. Partly, by the fact that the distribution on its way to the origin of the tension is appropriate to the intensity of the incoming information, the subsequently passed parts are appropriately stimulated. The effect is of course different in the root and in the sprout.

Partly, the local reconstruction happens, e.g. by a stimulation a new bifurcation is induced in the root just near places with the highest successfulness.

\subsection{The Control in the Sprout}

The principle is similar, but the working agent is different, e.g. cytokinin in connection with auxin. Accompanying cells monitor the tension on the boundary and depending on it; an information about its distribution comes into the phloem, appropriately aggregated. Auxin outputs from the phloem until getting into the parenchyma of the leaf. The influence happens here, for news in two directions. Firstly in a manner to augment the intensity in the area with successful inputs, it means an adequate regulation of stomas and of the structure of the spongy mesophyll for the appropriate income of the air. As a result the regulation of the income from the xylem probably happens. Thus the sequel of all this is the fact that parts with better conditions for the photosynthesis, e.g. better illumination, are more stimulated and vice versa.

The growth of the boundary itself is influenced as a sequel of the distribution of the working agent on the boundary, and thus the stable reconstruction happens depending on the successfulness, similarly as in the root.

\subsection{Column}

In the process of the processing of the tension a functional column of cells arises in the plant. At the beginning the information about the tension goes toward the phloem by the way of probably intracellular filamentary paths.
An important moment happens on the border of the phloem. This is the place where on one side the working agent—e.g. auxin—moves physically, it means in sieves, and on the other side is guided by filamentary structures inside of full-valued cells. It moves toward them in connection with the activity connected with the transfer of information in the opposite direction. Thus the column is formed by a group of cells realizing both kinds of these events - the way "up" and the way "down."

A way is chosen on the border of the phloem, toward the place with higher tension.

\section{Conclusion}

The plant as whole is controlled from two points of view. Firstly, it concerns the overall state on the boundary. The goal of the control is the state, when the tension is minimal. It is a state, when do not exist quite different influences from the environment side by side, but when the transition is gradual. This is reached by a control, where new analogical inputs are formed beside the maximally successful inputs and the less successful inputs are damped.

For this sake there is a mediator serving as an indicator of this state of tension and of its volatility on the boundary and is used in the control as a criterion of the overall satisfaction of the plant.

The second point of view is a view of the overall successfulness of cells. The energy and other substances are directed into successful areas of regeneration by a control with an analogical strategy as the control of the boundary. It stems from the maximally possible aggregation of information about the successfulness, and the goal is the aggregation over the plant as whole. And this is the second criterion, describing the overall satisfaction of cells.

\section{References}

[1] Vlcek, M. 2008. "Functioning of the Object." unpublished material.

[2] Vlcek, M. 2015. "Firm without Subordinations." American Journal of Economics, vol.5, nr.3. 
[3] Vlcek, M. 2015. "A Family-the Tension and the Satisfaction." non published material.

[4] Vlcek, M. 2015. "Philosophy of the Human Organism." non published material.

[5] Vlcek, M. 2010. "Movers of the Economy-Model of Interactions." in Developments in Economic Theory and Policy proceedings, Bilbao, Spain.

[6] Vlcek, M. 2014. "New Object Discovered through the
Stock Market." Presented at the International Conference on Economics and Finance Research, Seoul, South Korea.

[7] Vlcek, M. 2015. "Society and Its Way to the Happiness." American Journal of Economics 5 (4): 405-8.

[8] Vlcek, M. 2015. "Relations among American Firms Allow the Rise of a New Object." American Journal of Economics 5 (5): 423-7. 